\title{
Blood monocytes from mammary tumor-bearing mice: Early targets of tumor-induced immune suppression?
}

\author{
RAUL CASO $^{1}$, RISSET SILVERA ${ }^{2}$, ROBERTO CARRIO ${ }^{2}$, VIYAYA IRAGAVARAPU-CHARYULU ${ }^{3}$, \\ RUBEN R. GONZALEZ-PEREZ ${ }^{4}$ and MARTA TORROELLA-KOURI ${ }^{1,2}$
}

\author{
${ }^{1}$ Department of Microbiology and Immunology, University of Miami School of Medicine, ${ }^{2}$ Sylvester Cancer Center, \\ Miami, FL 33136; ${ }^{3}$ Department of Biomedical Sciences, Florida Atlantic University, Boca Raton, FL 33431; \\ ${ }^{4}$ Departments of Microbiology, Biochemistry and Immunology, Morehouse School of Medicine, Atlanta, GA 30310, USA
}

Received June 7, 2010; Accepted July 23, 2010

DOI: 10.3892/ijo_00000740

\begin{abstract}
We have previously shown that peritoneal macrophages from mice bearing advanced D1-DMBA3 mammary tumors are impaired in their inflammatory functions but are not alternatively activated either and are less differentiated than the ones from normal mice. However, little is known about whether similar defects exist in their precursor stages as blood monocytes. We examined if blood monocytes from mammary tumor-bearing mice are already altered in their activation profiles before becoming macrophages and whether they correspond to inflammatory or resident monocyte subtypes. Much effort is currently devoted to reversing macrophage adverse traits in tumor hosts; as these cells reside within tissues, access is limited. Blood monocytes could be better targeted and manipulated by less invasive means. In the present study, mononuclear cells were isolated from whole blood of D1-DMBA-3 mammary tumor-bearing and normal BALB/c mice and CD $115^{+}$monocytes were analyzed. Our results show that there is an increase in circulating monocytes in tumor hosts; these monocytes exhibit a reduced expression of several myeloid differentiation markers such as CD115, F4/80, CD68 and CD11b. Moreover, downregulation of MHC II, CD62L and the proangiogenic marker Tie-2 are observed in these cells, whereas $\mathrm{Gr}-1$ and Ly6C are upregulated. Furthermore, gene microarray analysis performed for the first time in blood monocytes from tumor hosts indicates that they express a mixture of pro-inflammatory and antiinflammatory cytokines and chemokines. Interestingly, CCR2 and CX3CR1, which are crucial in monocyte definition as inflammatory or resident, respectively, are both upregulated.
\end{abstract}

Correspondence to: Dr Marta Torroella-Kouri, University of Miami School of Medicine, P.O. Box 016960 (R-138), Miami, FL 33101, USA

E-mail: mtorroella@med.miami.edu

Key words: monocytes, macrophages, inflammation, immune suppression
Importantly, complement proteins are enhanced whereas nitric oxide production is decreased and there is no measurable arginase activity detected in these cells. Collectively, our study represents the first comprehensive analysis of blood monocytes from tumor-bearing mice; we conclude that these cells are neither completely inflammatory nor suppressive and are less differentiated, similar to the macrophages they later become.

\section{Introduction}

Inflammation and immune suppression are two opposing immune responses linked in different ways to cancer: while earlier stages of tumor development are associated with chronic inflammation (1), later stages of tumor progression are characterized by tumor-induced immune suppression $(2,3)$. Macrophages are key players of the inflammatory response and exhibit significant roles in the different stages of tumorigenesis. Their release of mutation-inducing free radicals (such as nitrogen and oxygen intermediates) as part of their cytotoxic/inflammatory response contributes to tumor initiation. Moreover, macrophages also play important roles in tumor progression by secreting factors that promote angiogenesis, invasion, extracellular matrix remodeling and metastasis. While tumor development is modulated by macrophages, the function of macrophages is likewise altered by the tumor resulting in macrophages that exhibit immune suppressive traits thereby contributing to tumor progression (4).

Circulating blood monocytes are the precursors of tissue macrophages. Monocytes originate in the bone marrow from a common myeloid progenitor shared with polymorphonuclear leukocytes (5). Monocytes are released into the peripheral blood where they circulate before entering tissues and replenishing tissue macrophage populations (6). Monocytes play an important role in development and homeostasis via removal of apoptotic cells and scavenging of toxic compounds (7). They constitute a group of immune effector cells, equipped with chemokine and adhesion receptors which mediate their migration from blood to tissues during infection or homeostasis (8). Monocytes show the ability to recognize lipids and various microorganisms, and once stimulated they can 
produce large quantities of reactive-oxygen species (ROS), complement factors, prostaglandins, nitric oxide (NO), diverse cytokines and vascular endothelial growth factor (VEGF) (7). Phenotypic characterization of monocytes found in mice bearing infections has allowed the identification of two discrete monocyte subsets (7-11). These two populations have been described as being $\mathrm{CD} 115^{+} \mathrm{Ly} 6 \mathrm{C}^{\mathrm{hi}} \mathrm{CX}_{3} \mathrm{CR} 1^{\text {lo }} \mathrm{CCR} 2{ }^{+} \mathrm{CD} 62 \mathrm{~L}^{+}$ inflammatory monocytes, and CD $115^{+} \mathrm{Ly}_{6 \mathrm{C}}{ }^{\mathrm{lo}} \mathrm{CX}_{3} \mathrm{CR} 1{ }^{\text {hi } C C R} 2$ CD62L- resident monocytes (12). Inflammatory monocytes are selectively recruited to inflamed tissues and lymph nodes and are the main producers of TNF- $\alpha$ and IL-1. However, the resident monocytes have a longer half-life and exhibit a constitutive long-range, crawling on the luminal side of the endothelium within most blood vessels (7).

Although extensive data have been collected describing the functions of monocytes during inflammation as a result of infection, evidence is lacking regarding the characteristics of monocytes in the context of tumor burden. We have previously shown that peritoneal macrophages from mice bearing advanced mammary tumors are defective in their inflammatory response $(13,14)$. In the present work we address the challenge of characterizing a cell population present in low percentages in the blood, as monocytes are, and performed a broad analysis of these cells in tumor-bearing mice as compared to their normal counterparts. We investigated whether $\mathrm{CD} 115^{+}$blood monocytes from tumor-bearing mice are defective prior to differentiating into macrophages within tissues. In this regard, our data corroborated that tumor hosts exhibit an alteration in myelopoiesis, leading to an accumulation of monocytes in the blood, as has been described by us and others $(14,15)$. We also provide evidence that $\mathrm{CD} 115^{+}$blood monocytes from tumor-bearing mice overexpress complement (such as $\mathrm{C} 3$ and $\mathrm{C} 5 \mathrm{a}$ receptor), display lower levels of key myeloid differentiation markers, downregulate $\mathrm{MHC}$ II, CD62L and the proangiogenic marker Tie-2, yet upregulate Gr-1 and Ly6C. Moreover, they are also defective in their production of $\mathrm{NO}$ and lack arginase activity. All together, we conclude that blood monocytes from mammary tumor bearers, as previously shown with macrophages from mammary tumor bearers (14), are neither completely pro-inflammatory nor anti-inflammatory and are not phenotypically part of a group of undifferentiated heterogeneous myeloid cells that have been described in tumor-bearing mice, the myeloid-derived suppressor cells (MDSCs) $(16,17)$. The exposure of monocytes or peritoneal macrophages to circulating tumor factors may be responsible for such alterations in these cells from tumor hosts.

\section{Materials and methods}

Animals and tumor. Female BALB/c mice of 10-12 weeks of age were used. In all experiments, tumor-bearing mice and their normal controls were age matched. The D1-DMBA-3 mammary adenocarcinoma (18) was maintained and used as previously described (19). The Institutional Animal Care and Use Committee (IACUC) approved all our animal experiments.

Cell isolation and purification. Mice bearing 4-week tumors and their normal controls were euthanized by $\mathrm{CO}_{2}$. To isolate blood monocytes, peripheral blood was drawn via cardiac puncture using heparin sodium (Abraxis Pharmaceutical Products, Schaumburg, IL) as an anti-coagulant. Mononuclear cells were purified by density gradient centrifugation using the Ficoll-Paque Premium 1.084 medium (GE Healthcare Bio-sciences, Piscataway, NJ) following the manufacturer's instructions. Leukocytes and platelets in the Buffy coat constitute $<1 \%$ of total blood and mouse monocytes in the Buffy coat constitute only $2-5 \%$ of blood cells. Thus, for the purposes of the phenotypic analysis using flow cytometry, the blood of 3 tumor-bearing and 3 control mice were respectively pooled and examined, while for the microarrays, NO and arginase studies the blood of 25 tumor-bearing mice and 80 normal mice were, respectively, pooled for each experiment to obtain enough monocytes. Thioglycollate-elicited peritoneal macrophages were isolated as previously described (20). Viable cell numbers were determined using trypan blue (Thermo Scientific) and cells were counted using a hemacytometer.

Flow cytometry. Mononuclear cells and peritoneal macrophages from normal and tumor-bearing mice were washed and counted. Fc receptors were blocked using mouse CD16/32 antibody (eBioscience, San Diego, CA) and the following antibodies were used: F4/80-PE-Cy7 and Tie-2 PE (both from BioLegend, San Diego, CA), Ly6C-biotin (BD Pharmingen, San Jose, CA), CD11b-FITC, CD115-PE, Gr-1-APC, CD62Lbiotin, and MHC II-Alexa Fluor 700 (all from eBioscience). Strep-Pacific Blue (eBioscience) was utilized to label biotinylated antibodies. Intracellular staining with CD68Alexa Fluor 700 (AbD Serotec, Raleigh, NC) was performed according to the manufacturer's instructions. For analysis of the cell surface expression of C5aR by blood monocytes, cells were sequentially incubated with rabbit polyclonal anti-mouse C5aR (BD Pharmingen) and fluorescein isothiocyanate-conjugated anti-rabbit IgG (R\&D Systems, Minneapolis, MN). Data were acquired on an LSRII (BD Biosciences, San Jose, CA) and analyzed with BD FACSDiva Software (BD Biosciences).

Microarray gene expression profiling. Blood monocytes from normal and tumor-bearing mice were analyzed by fluorescence activated cell sorting (FACS). Monocytes were identified based on their FSC-A ${ }^{\text {hi }}$ SSC- $A^{\text {hi }}$ profile and their expression of CD115 using the BD FACSAria I sorter (BD Biosciences). A total of $10^{6}$ sorted cells were resuspended in complete RPMI [RPMI-1640 containing 10\% FCS, 2 mM of L-glutamine with $100 \mathrm{U}$ of penicillin and $100 \mu \mathrm{g} / \mathrm{ml}$ of streptomycin (all from Hyclone, UT)] and incubated with or without lipopolysaccharide (LPS) from Escherichia coli 055:B5 (Sigma-Aldrich, St. Louis, MO) at a concentration of $10 \mu \mathrm{g} / \mathrm{ml}$ in a $\mathrm{CO}_{2}$ chamber at $37^{\circ} \mathrm{C}$ for $2 \mathrm{~h}$. RNA was extracted using the Array Grade Total RNA Isolation Kit (GA-013; SABiosciences, Frederick, MD). Total RNA was quantified using the Nanodrop ND-100 (Nanodrop Technologies, Inc., Wilmington, DE). Equal amounts of labeled probe were hybridized to Oligo GEArray Mouse Chemokines and Receptors (OMM-022), Mouse Common Cytokines (OMM021), and Mouse Inflammatory Cytokines and Receptors (OMM-011; SABiosciences) arrays, following the manufacturer's instructions. Densitometry was performed, normalization 
was carried out using $\beta$-actin gene expression and heat maps were generated to indicate the gene expression levels.

Nitric oxide production. Sorted CD $115^{+}$monocytes from normal and tumor-bearing mice were plated $\left(3 \times 10^{5}\right.$ cells $/ 96-$ well flat-bottom) and incubated with and without $10 \mu \mathrm{g} / \mathrm{ml}$ of LPS in complete RPMI for $48 \mathrm{~h}$. Nitrite $\left(\mathrm{NO}_{2}{ }^{-}\right)$concentration determined in the cell supernatant served as a reflection of NO production and was measured using the colorimetric Griess reaction (21) as previously described (22).

Functional arginase assay. Sorted $\mathrm{CD} 115^{+}$monocytes and peritoneal macrophages from normal and tumor-bearing mice were plated $\left(3 \times 10^{5}\right.$ cells/96-well flat-bottom) and incubated in complete RPMI with or without $10 \mu \mathrm{g} / \mathrm{ml}$ of LPS. After $48 \mathrm{~h}$, cells were washed with PBS, and $50 \mu \mathrm{l}$ of lysis buffer [10 $\mathrm{ml} \mathrm{H}_{2} \mathrm{O}, 1$ tablet Complete Mini EDTA (ethylenediaminetetraacetic acid)-free protease inhibitor (Roche, Mannheim, Germany), and 0.1\% Triton X-100 (SigmaAldrich)] was added to each well. Cells were incubated at $37^{\circ} \mathrm{C}$ for $30 \mathrm{~min}$, and lysates were transferred to microfuge tubes. Fifty $\mu \mathrm{l}$ of $25 \mathrm{mM}$ Tris-HCl (Sigma-Aldrich) and $10 \mu \mathrm{l}$ of $2 \mathrm{mM} \mathrm{MnCl}_{2}$ (Alfa Aesar, Ward Hill, MA) were added to each sample. Samples were then heated at $56^{\circ} \mathrm{C}$ for $10 \mathrm{~min}$, and $100 \mu 1$ of $500 \mathrm{mM}$ L-arginine (Alfa Aesar), $\mathrm{pH}$ 9.7, was added. Arginine hydrolysis was stopped after $30 \mathrm{~min}$ of incubation at $37^{\circ} \mathrm{C}$, with $800 \mu \mathrm{l}$ of acid solution $(1: 3: 7,96 \%$ $\left.\mathrm{H}_{2} \mathrm{SO}_{4}: 85 \% \mathrm{H}_{3} \mathrm{PO}_{4}: \mathrm{H}_{2} \mathrm{O}\right)$. Forty $\mu \mathrm{l}$ of $4 \% \alpha$-isonitrosopropiophenone (Sigma-Aldrich) in $100 \%$ ethanol were added to precipitate the urea by-product. Samples were heated at $100^{\circ} \mathrm{C}$ for $30 \mathrm{~min}$. Optical density was measured at $540 \mathrm{~nm}$. A standard curve was performed by running samples of urea (Sigma-Aldrich) in lysis buffer parallel to cell lysates.

Statistical analysis. Error bars represent the standard error of the mean (SEM), and all P-values were from two-sided t-test. Single asterisk denotes $\mathrm{P}<0.05$, two asterisks indicate $\mathrm{p}<0.01$, and three asterisks designate $\mathrm{p}<0.001$.

\section{Results}

Selection based on CD115 rather than on F4/80 identifies monocyte populations with higher purity. To better isolate monocytes, F4/80 and CD115 antibodies (both expressed by cells of the myeloid lineage) were used. To this end, monocytes were first identified as FSC-A ${ }^{\text {hiSSC}}-\mathrm{A}^{\text {hi }}$ in contrast to the smaller $\mathrm{T}$ - and B-lymphocyte population found in the mononuclear cell fraction obtained after density gradient centrifugation. Using F4/80 to isolate monocytes revealed that $29 \%$ of the FSC-A ${ }^{\text {hi SSC-A }} \mathrm{A}^{\text {hi }}$ population expressed this marker (Fig. 1A). However, upon analysis of the purity of this population, cells expressing CD3 and CD19 (markers used to characterize T-cells and B-cells, respectively) were also observed, $\sim 50 \%$ expressed CD3 while $35 \%$ expressed CD19, Fig. 1B). On the other hand, when monocytes were isolated using CD115 (Fig. 1A), roughly $26 \%$ of the FSC$\mathrm{A}^{\text {hiSSC}}-\mathrm{A}^{\text {hi }}$ population expressed this marker and the purity was much higher with $<6 \%$ expressing CD3 and $<1 \%$ expressing CD19 (Fig. 1B).

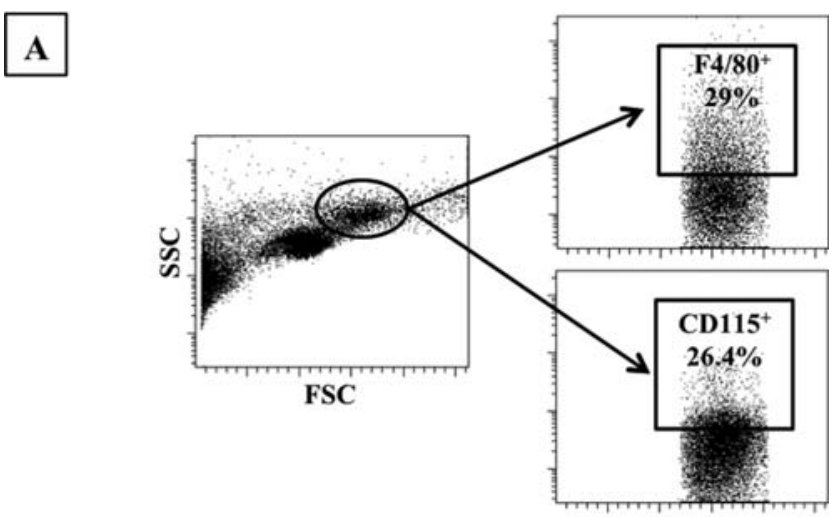

B

Population Purity

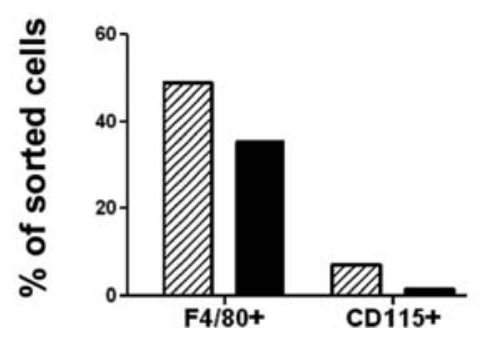

Figure 1. Selection using CD115 marker results in pure monocyte populations The purity of the mononuclear cells extracted from peripheral blood of mice was studied. (A) FSC-A ${ }^{\text {hi SSC-A }}{ }^{\text {hi }}$ population was gated and studied for the individual expression of F4/80 and CD115 in the mononuclear cell fraction obtained after density gradient centrifugation of peripheral blood of tumor-bearing mice. (B) Cell sorting using CD115 antibody exhibits a lower percentage of cells expressing $\mathrm{CD}^{+}$and $\mathrm{CD} 19^{+}$as compared to selection with F4/80. Figures represent one of five different experiments with similar results.

Tumor-bearing mice have increased numbers of CD115+ circulating blood monocytes downregulating myeloid differentiation markers and upregulating Ly6C and Gr-1. In previous studies we demonstrated an expansion in bone marrow-derived myeloid progenitors in tumor bearers (14). In the present study we investigated whether these enhanced numbers of bone marrow progenitors resulted in increased amounts of blood monocytes in tumor hosts. To this end monocytes from normal and tumor-bearing mice were studied. Upon selection of the monocyte population among FSC$\mathrm{A}^{\text {hiSSC- }} \mathrm{A}^{\text {hi }}$ cells, only the cells expressing CD115 were analyzed. In comparison with their control counterparts, tumor-bearing mice had significantly $\left.{ }^{* * *} \mathrm{P}=0.0004\right)$ higher percentages of circulating $\mathrm{CD} 115^{+}$monocytes in the blood, corresponding to a 9-fold increase compared to those found in the blood of control mice (Fig. 2A). When studying the expression of differentiation markers, our data showed a downregulation in the mean fluorescence intensity (MFI) of CD115 ( $\left.{ }^{*} \mathrm{P}=0.0486\right), \mathrm{CD} 11 \mathrm{~b}\left({ }^{*} \mathrm{P}=0.0205\right), \mathrm{F} 4 / 80\left({ }^{*} \mathrm{P}=0.0482\right)$, and CD68 ( $\left({ }^{*} \mathrm{P}=0.0477\right)$ on $\mathrm{CD} 115^{+}$-selected blood monocytes from tumor-bearing mice as compared to normal mice (Fig. 2). Furthermore, flow cytometric analysis showed a substantial increase in the expression of these myeloid differentiation markers (in terms of MFI) in resident peritoneal macrophages 


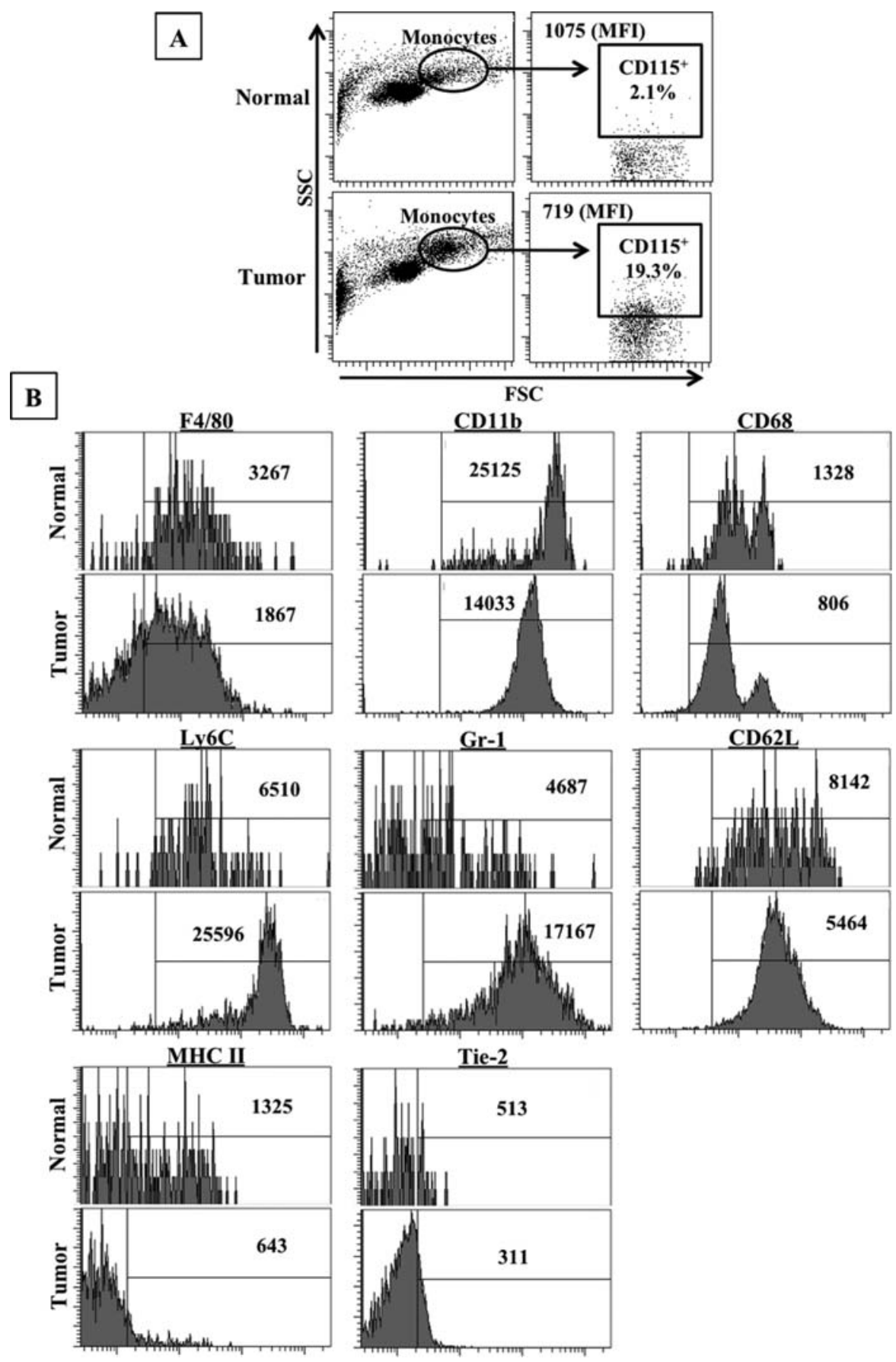

Figure 2. Monocytes from tumor-bearing mice are more numerous and less differentiated. (A) Flow cytometric analysis of the mononuclear cell fraction obtained after density gradient centrifugation of peripheral blood shows the FSC and SSC profiles of blood monocytes from normal and tumor-bearing mice (top and bottom left). Analysis of CD115 expression by monocyte population (FSC- ${ }^{\text {hisSC-A }} \mathrm{A}^{\text {hi }}$ ) obtained from the mononuclear cell fraction of peripheral blood from tumor-bearing mice, bottom right, and normal mice, top right (percent of cells gated and mean fluorescence intensity, MFI, shown). (B) Analysis of the expression of F4/80, CD11b, CD68, Ly6C, Gr-1, CD62L, MHC II, and Tie-2 by CD115+ blood monocytes obtained from normal, top, and tumor-bearing mice, bottom, (MFI shown). Data represent one of five different experiments with similar results.

from both types of mice as compared with their corresponding monocyte counterparts (data not shown). Moreover, our results also showed an upregulation in the level of expression of Gr-1 $\left({ }^{* *} \mathrm{P}=0.0058\right)$ and Ly6C $\left({ }^{*} \mathrm{P}=0.0386\right)$ on the surface of CD $115^{+}$blood monocytes from tumor-bearing mice as compared with their expression levels on blood monocytes from normal mice (Fig. 2B).
Circulating CD115+ blood monocytes from tumor-bearing mice downregulate L-selectin, Tie-2, and MHC II. We also compared the capacity of CD $115^{+}$blood monocytes from tumor-bearing mice to express other markers by flow cytometry. Analysis of the expression of CD62L (L-selectin) by $\mathrm{CD} 115^{+}$blood monocytes indicates that its expression on the cell membrane is downregulated in monocytes from tumorbearing mice (Fig. 2B; ${ }^{\mathrm{P}}=0.0323$ ). Moreover, the expression of 


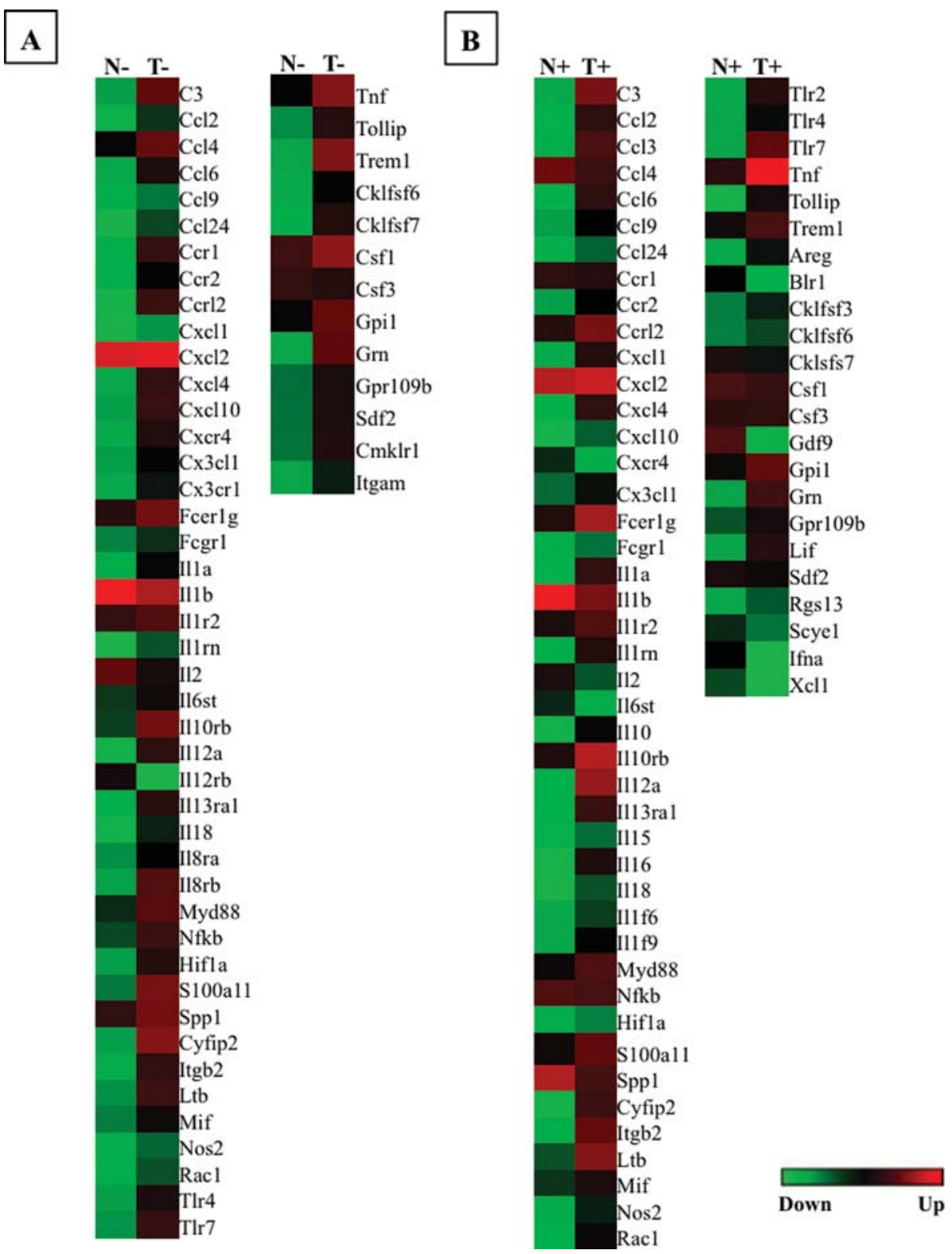

Figure 3. Heat maps of transcripts expressed by CD115+ blood monocytes. (A) Gene transcripts constitutively expressed by sorted CD115+ blood monocytes from normal $\left(\mathrm{N}^{-}\right)$and tumor-bearing $\left(\mathrm{T}^{-}\right)$mice after $2 \mathrm{~h}$ of incubation in media only. (B) Gene transcripts expressed by sorted CD115+ blood monocytes from normal $\left(\mathrm{N}^{+}\right)$and tumor-bearing $\left(\mathrm{T}^{+}\right)$mice activated for $2 \mathrm{~h}$ with LPS $(10 \mu \mathrm{g} / \mathrm{ml})$. Figure represents one of two different experiments with similar results using three different gene expression pathways (chemokines and receptors, inflammatory cytokines and receptors, and common cytokines). Color codes indicate high expression (red) and low expression (green).

Tie-2 in these cells was also decreased (Fig. $2 \mathrm{~B} ;{ }^{* *} \mathrm{P}=0.0019$ ). Tie-2 is the receptor for angiopoietin with CD115+Tie2 ${ }^{+}$ monocytes describing a distinct lineage of proangiogenic monocytes required for the promotion of tumor growth $(23,24)$. In addition, $\mathrm{CD} 115^{+}$monocytes from tumor-bearing mice exhibit a downregulation in MHC II expression (Fig. 2B; ${ }^{*} \mathrm{P}=0.0122$ ).

CD115+ blood monocytes from tumor-bearers express the transcript for C3 complement and a mixture of pro-inflammatory and anti-inflammatory genes. We hypothesized that the increased number of blood monocytes in tumor bearers also exhibit altered patterns of chemokine and cytokine expression. To begin to elucidate this, mRNA expression of chemokines, cytokines, and their respective receptors were examined using microarray technology. Analysis of the constitutive (Fig. 3A) and LPS-induced (Fig. 3B) expression of several genes from three different signal transduction pathways by $\mathrm{CD} 115^{+}$blood monocytes from normal and tumor-bearing mice revealed a number of upregulated and downregulated transcripts. Monocytes from tumor-bearing mice differed from their normal counterparts in regards to the presence of two main groups of transcripts. One of these groups is involved in the inflammatory response: monocytes from tumor-bearers showed an enhanced expression of proinflammatory molecules such as IL-12 $\alpha$ (IL-12p35), C3 which is a member of the complement system, CXCL10, CCL6, TNF $\alpha$, TLR7, IL-1 $\alpha$, IL-18, CCR2, TLR4 and CCL2 
Table I. Transcripts expressed in CD115+ blood monocytes sorted from tumor-bearing mice. ${ }^{\mathrm{a}}$

\begin{tabular}{|c|c|c|c|}
\hline Gene name & Gene symbol & $\begin{array}{c}\text { Constitutive expression } \\
\text { (fold change) }\end{array}$ & $\begin{array}{l}\text { LPS-induced expression } \\
\text { (fold change) }\end{array}$ \\
\hline
\end{tabular}

\begin{tabular}{|c|c|c|c|}
\hline \multicolumn{4}{|l|}{ Pro-inflammatory response } \\
\hline Interleukin 12 alpha & $I l 12 a$ & 61.99 & 137.39 \\
\hline Complement component 3 & C3 & 39.51 & 78.56 \\
\hline Chemokine (C-X-C motif) ligand 10 & Cxcl10 & 33.37 & 15.03 \\
\hline Chemokine (C-C motif) ligand 6 & Ccl6 & 22.58 & 71.86 \\
\hline Tumor necrosis factor & $\operatorname{Tnf}$ & 19.57 & 4.05 \\
\hline Toll-like receptor 7 & $T l r 7$ & 18.83 & 48.61 \\
\hline Interleukin 1 alpha & Illa & 16.92 & 74.48 \\
\hline Interleukin 18 & Ill18 & 16.83 & 19.29 \\
\hline Chemokine (C-C motif) receptor 2 & Ccr2 & 15.27 & 7.78 \\
\hline Toll-like receptor 4 & $\operatorname{Tlr} 4$ & 15.09 & 9.12 \\
\hline Chemokine (C-C motif) ligand 2 & Ccl2 & 13.57 & 68.71 \\
\hline Toll interacting protein & Tollip & 11.55 & 65.14 \\
\hline Myeloid differentiation primary response gene 88 & Myd88 & 6.89 & 3.23 \\
\hline Nuclear factor kappa light chain & $N f k b$ & 6.72 & - \\
\hline Nitric oxide synthase 2 , inducible, macrophage & $\operatorname{Nos} 2$ & 5.37 & 11.28 \\
\hline Interferon alpha & Ifna & - & -57.03 \\
\hline Toll-like receptor 2 & $\operatorname{Tlr} 2$ & - & 30.16 \\
\hline Interleukin 1 beta & $I l 1 b$ & -2 & -2.13 \\
\hline Interleukin 12 receptor, beta & $I l 12 r b$ & -59.29 & - \\
\hline \multicolumn{4}{|l|}{ Anti-inflammatory response } \\
\hline Interleukin 10 & Illo & - & 35.71 \\
\hline Interleukin 10 receptor, beta & IllOrb & 9.83 & 3.87 \\
\hline Interleukin 1 receptor antagonist & Illrn & 17.64 & 44.33 \\
\hline Interleukin 1 receptor, type II & Illr2 & - & 2.28 \\
\hline Interleukin 13 receptor, alpha 1 & Ill3ral & 45.22 & 68.43 \\
\hline Chemokine (C-C motif) ligand 24 & Ccl24 & 17.14 & 9.68 \\
\hline \multicolumn{4}{|l|}{ Other genes of interest } \\
\hline Cytoplasmic FMR1 interacting protein 2 & Cyfip2 & 47.38 & 134.08 \\
\hline Chemokine (C-X-C motif) receptor 4 & Cxcr 4 & 25.63 & -13.09 \\
\hline Hypoxia inducible factor 1 , alpha subunit & Hifla & 18.34 & 4.31 \\
\hline Chemokine (C-X3-C motif) receptor 1 & $C \times 3 c r 1$ & 8.98 & - \\
\hline Chemokine (C-X3-C motif) ligand 1 & Cx3cll & 3.29 & 2.21 \\
\hline
\end{tabular}

${ }^{a}$ The fold changes were obtained by comparison of $\mathrm{CD} 115^{+}$blood monocytes from tumor-bearing mice with control CD115+ blood monocytes from control mice after standardizing the individual densities with actin.

(Table I). In addition, a few of the other pro-inflammatory genes that were upregulated in monocytes from tumor bearers include MyD88, Tollip, NFкB1 which encodes for NFкBp50 protein, and inducible nitric oxide synthase 2 (iNOS2). Interestingly, we found downregulation in the expression of pro-inflammatory IL-1ß, IFN $\alpha$ and IL-12R 3 . The other main group of genes that were significantly altered in monocytes from tumor-bearing mice compared to their normal counterparts concern the anti-inflammatory response and ultimately with signals involved with immunosuppression (Table I). Moreover, monocytes from tumor-bearing mice upregulate the transcripts encoding for cytokine IL-10 and its receptor IL10Rß. The mRNAs for IL-1 receptor antagonist, IL-3R $\alpha 1$ and the chemokine CCL24 are also elevated.

C5 a receptor protein is significantly upregulated in monocytes from tumor bearers. Our finding that the $\mathrm{C} 3$ complement transcript is highly upregulated in monocytes from tumor bearers (both constitutively and after LPS activation) is significant. Therefore, we further examined whether C5a receptor, which is normally induced by $\mathrm{C} 3$, was also enhanced in monocytes from tumor bearers. Flow cytometry results 


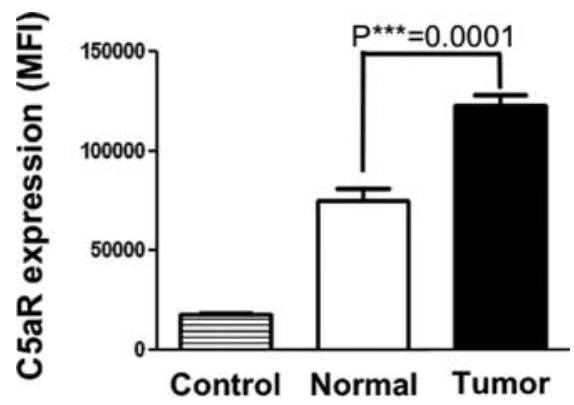

Figure 4. C5a receptor is upregulated in monocytes from tumor bearers Flow cytometry analysis of the expression of $\mathrm{C} 5 \mathrm{aR}$ by $\mathrm{CD} 115^{+}$blood monocytes from normal and tumor-bearing mice; control represents CD115 monocytes from normal mice that were not stained with C5aR, MFI shown. Figure represents one of three different experiments with similar results.

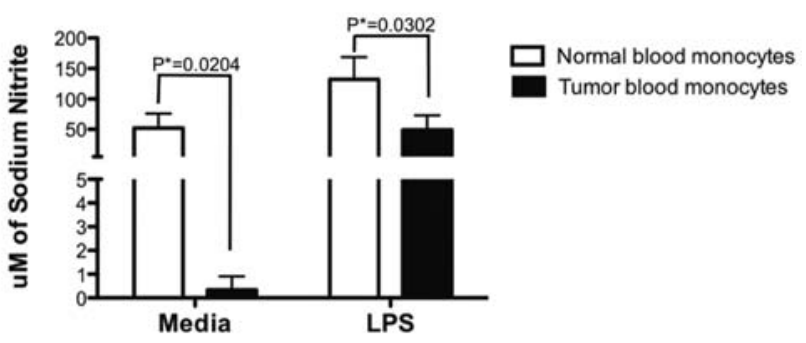

Arginase Activity

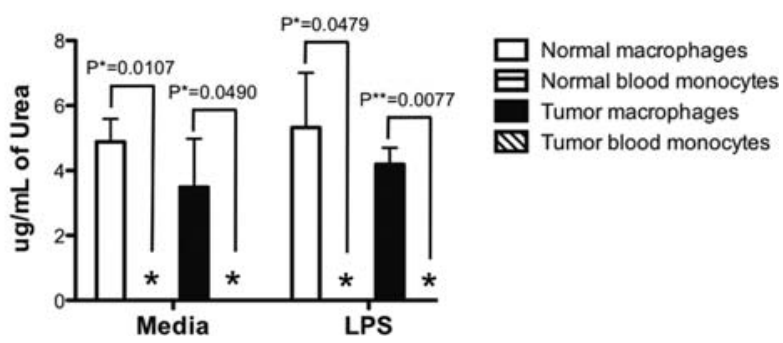

Figure 5. Blood monocytes from tumor-bearers exhibit a defective production of nitric oxide (NO) compared to normal mice and no measurable arginase activity. (A) Sorted CD $115^{+}$blood monocytes from normal and tumor-bearing mice were incubated with and without LPS $(10 \mu \mathrm{g} / \mathrm{ml})$ for $48 \mathrm{~h}$. Results represent one of five independent experiments with similar results. Bars represent the mean amount of $\mathrm{NO}^{-}$detected in the cell supernatants in $\mu \mathrm{M}$. (B) Determination of the amount of urea detected in $\mu \mathrm{g} / \mathrm{ml}$ as an indication of arginase activity within CD $115^{+}$blood monocytes from normal and tumorbearing mice after being cultured in media or LPS $(10 \mu \mathrm{g} / \mathrm{ml})$ for $48 \mathrm{~h}$ Thioglycollate-elicited peritoneal macrophages from normal and tumorbearing mice were included as controls and cultured likewise. Data represent one of five different experiments with similar results.

showed that $\mathrm{C} 5 \mathrm{aR}$ is highly expressed $\left({ }^{* * *} \mathrm{P}=0.0001\right)$ on the surface of $\mathrm{CD} 115^{+}$blood monocytes from tumor-bearing mice when compared to those observed in normal mice (Fig. 4).
Monocytes from tumor-bearers produce low levels of $\mathrm{NO}$ compared to their normal counterparts and lack arginase activity. To further investigate the inflammatory function of monocytes from tumor bearers, the levels of NO were assessed in both cell types. We studied the production of NO in both cell types. Monocytes from tumor bearers produced very low quantities of NO compared to their normal counterparts when cultured in media only (Fig. 5A; ${ }^{*} \mathrm{P}=0.0204$ ). However, they were induced to produce higher levels of NO after LPS activation, which were still significantly lower than the amounts produced by their LPS-activated normal counterparts (Fig. 5A; ${ }^{*} \mathrm{P}=0.0302$ ). Moreover, monocytes from either normal or tumor-bearing mice do not exhibit measurable constitutive or LPS-induced arginase activity, in contrast to peritoneal elicited macrophages from either animal type, which display similar amounts of arginase activity both constitutively or upon LPS activation (Fig. 5B).

\section{Discussion}

The bona fide blood monocyte population found in mice bearing advanced tumors has not been extensively addressed. Due to the association between chronic inflammation and cancer development (1), we sought to examine whether blood monocytes from mice bearing advanced D1-DMBA3 mammary tumors might correspond to inflammatory monocytes or instead displayed an immune suppressive phenotype. We used the myeloid markers F4/80 and CD115 (receptor for macrophage colony-stimulating factor, M-CSF) to sort monocytes from mononuclear cells separated from whole blood of mice, yet our results show that they do so with varying levels of purity. Based on the higher purity obtained when using CD115 as opposed to F4/80 we decided to use CD115 to purify monocytes from the Ficoll-Paque-derived mononuclear cell fraction from the blood of normal and tumor-bearing mice. Our analysis of the blood monocyte population in tumor-bearing mice shows a substantial increase in the percentage of circulating CD $115^{+}$monocytes (19.3 vs $2.1 \%$ ). This increase may be due to enhanced myelopoiesis within the bone marrow of tumor hosts and has been associated with the action of tumor factors $(15,25)$. However, we have shown in the present mammary tumor model that an augment in marrow myelopoiesis in tumor hosts may also be a direct result of increased macrophage apoptosis in the peritoneum of tumor-bearing mice and thus part of a compensatory homeostatic mechanism (14). Cells from the myeloid lineage express key lineage markers such as CD115, CD11b, F4/80 and CD68, and the elevated expression of these molecules is considered indicative of myeloid differentiation (26-29). Phenotypic analysis comparing MFIs of these differentiation markers on $\mathrm{CD} 115^{+}$blood monocytes from normal and tumorbearing mice revealed a downregulation in the MFIs of CD115, CD11b, F4/80, and CD68 in monocytes from tumor hosts, indicating that they exhibit a more immature phenotype compared to those from normal mice. We compared the levels at which monocytes and peritoneal macrophages from normal or tumor-bearing mice display these myeloid differentiation markers. Our results suggest that, as expected, macrophages from each group of mice express higher levels of differentiation markers than their corresponding monocyte precursors (data not shown). 
The phenotypic marker Gr-1 (mainly expressed by granulocytes) recognizes Ly6C and Ly6G molecules; $\mathrm{Gr}-1$ and Ly6C are cell surface proteins that have been described to be expressed by monocytes involved in the inflammatory response (30-32) and according to Auffray et al (7), the Gr-1 and Ly6C-specific antibodies label the same cells in the mouse blood. Our CD115+ blood monocytes from tumorbearers upregulated their expression of Gr-1 and Ly6C, indicating that they might be inflammatory monocytes (33). Importantly, Gr-1 and CD11b are simultaneously upregulated in MDSCs of mice. MDSCs are a heterogeneous group of immature myeloid cells that accumulate in different locations in tumor hosts and exhibit immune suppression capabilities, specifically by inhibiting $\mathrm{T}$ cell proliferation $(34,35)$. However, our data show that CD $115^{+}$blood monocytes from tumor bearers do not exhibit CD11b upregulation, instead they show a decreased expression of this marker, therefore CD115+ blood monocytes from our tumor hosts do not phenotypically correspond to MDSCs.

Phenotypic analysis also revealed that $\mathrm{CD} 115^{+}$blood monocytes from tumor bearers downregulate the expression of CD62L (L-selectin), an adhesion molecule that contributes to leukocyte adhesion and migration, essential for the recruitment of monocytes to the tissues and whose expression has been described on inflammatory monocytes $(7,36)$. In CD62L-deficient mice, the number of monocytes recruited following thioglycollate-induced peritonitis was reduced by more than $70 \%$ compared with wild-type mice (36). Thus, a decrease in the expression of CD62L in monocytes from tumor-bearers suggests that these cells may be unable to properly adhere to the endothelial wall and extravasate into the inflamed tissue indicating that they might be more of the resident type. Our results also show that $\mathrm{CD} 115^{+}$blood monocytes downregulate MHC II. Downregulation of MHC II by tumor cells is one of the mechanisms by which tumors induce immune suppression, evading the host's anti-tumor immune responses. Moreover, CD $115^{+}$blood monocytes from tumor-bearers do not upregulate the expression of Tie-2 suggesting that they may not be part of the group of Tie-2expressing monocytes (TEMs) described in tumor-bearing mice, with selective homing to the tumor site where they are required for angiogenesis (37).

Comparative gene expression profiling of blood monocytes from normal and tumor-bearing mice has not been addressed before. Using gene array analysis we examined the transcriptome of chemokines, chemokine receptors, cytokines and their receptors in blood monocytes from normal and tumor-bearing mice. Our data revealed that CD115+ blood monocytes from tumor hosts express a mixture of pro- and anti-inflammatory traits. Among the cytokines and signal transduction pathway mediators of an inflammatory response that are upregulated in monocytes from tumor hosts are the p35 chain (IL-12p35) of the pro-inflammatory and anti-tumor cytokine IL-12p70, IL-18, a cytokine that shares some of the properties of IL-12, TNF $\alpha$, a pro-inflammatory cytokine associated with tumor development, IL-1 $\alpha$, a pro-inflammatory cytokine that promotes angiogenesis by inducing VEGF synthesis and secretion; also TLR7, TLR4, MyD88, Tollip, NFkB1 and iNOS2. Interestingly, IL-12p35 is the gene that shows the highest expression (both constitutively and upon
LPS stimulation) in monocytes from tumor hosts, in contrast with peritoneal macrophages from the same mice which exhibit decreased mRNA levels of IL-12p35 upon LPS stimulation and almost no constitutive expression of the gene (13). Strikingly, the C3 member of the complement system was highly overexpressed by monocytes from tumor bearers. Complement proteins circulate in the blood and are important in the innate immune defense. These proteins are known to be produced mainly in the liver, but extra-liver sources of complement are produced by macrophages and monocytes. Our results showing that monocytes from tumor bearers over express $\mathrm{C} 3$ transcripts indicates that a significant portion of the complement molecules in the blood of tumor bearers may be provided by monocytes; these results are significant since complement proteins have been recently associated with tumor progression (38-41). The overexpression of C3 in monocytes from tumor bearers prompted us to examine the expression of the receptor for $\mathrm{C} 5 \mathrm{a}(\mathrm{C} 5 \mathrm{aR})$ on these monocytes; the tumor may be exploiting the complement system via the classical activation pathway using the high levels of $\mathrm{C} 3$ to generate more C5a which is known to attract pro-tumor MDSCs expressing C5aR, thus setting an immunosuppressive environment (39). This could result in a feedback loop where more recruitment of $\mathrm{C} \mathrm{aR}^{+}$bone marrow monocytes to the blood containing C5a may occur, and also to the tumor, where complement molecules may also be deposited. Interestingly, we found that $\mathrm{CD} 115^{+}$blood monocytes from tumor-bearing mice upregulate the expression of $\mathrm{C} 5 \mathrm{aR}$. C5aR induces suppression of an antitumor CD8 response associated with the recruitment MDSC into tumors and augments $\mathrm{T}$ cell-directed suppressive abilities (42), and pharmacological blockade of the C5a receptor considerably impairs tumor growth (39). Moreover, C5a has the ability to selectively suppress TLR4-mediated expression of IL-12 family members IL-12, IL-23, and IL-27, thus ultimately favoring the anti-inflammatory response over the pro-inflammatory response.

Among the pro-inflammatory chemokines that are upregulated in monocytes from tumor hosts are CCL2 and its receptor CCR2, also CXCL10 and CCL6. CCR2 is a major player in the recruitment and activation of circulating monocytes (43-45) and a marker of inflammatory monocytes. It is interesting to note that monocytes from tumor bearers downregulate CD62L but at the same time upregulate CCR2 and CX3CR1, all important factors directing in opposite ways the departure of monocytes from the blood vessels into the tissues. CCR2 and CX3CR1, which are the main chemokine receptors involved in monocyte classification as inflammatory or resident respectively, are both overexpressed in monocytes from tumor bearers, albeit at different levels, thus suggesting that the main monocyte population in the peripheral blood of mice bearing tumors is not characteristically resident nor inflammatory but a mixture of both. Our results further contribute to understanding the complexity of the in vivo environment; how monocytes ultimately operate is greatly complicated by tumor factors and depends on a multitude of interactions and synergisms within the tumor host.

The other group of genes whose expressions were significantly changed in monocytes from tumor-bearing mice is involved in the anti-inflammatory response. Monocytes from tumor hosts upregulate transcription of IL-10 and of its receptor 
IL10Rß, of IL-1 receptor antagonist, IL-3R $\alpha 1$ and chemokine CCL24, all immune suppressive intermediates that in different ways contribute to tumor progression. Interestingly, monocytes from tumor hosts upregulate the expression of both Hif1a and CXCR4 which have been found to modulate the recruitment of myeloid cells to hypoxic regions within the tumor microenvironment. Hifla has been found to be upregulated when cells are in a hypoxic environment (34). CXCR4 expression and chemotactic function in different myeloid populations is strongly increased through the activation of Hif1a (46). Furthermore, hypoxia strongly affects the chemokine system of monocytes/macrophages (47). Thus our results suggest that CXCR4-expressing monocytes from tumor-bearing mice may be recruited to the hypoxic environment of a progressing tumor to be differentiated into tumor-associated macrophages. Taken together, our microarray data suggest that CD115+ blood monocytes from tumor-bearing mice elude the rigid classification of pro-inflammatory or anti-inflammatory phenotypes but rather exhibit a mixture of these two opposite activation profiles.

In addition, we found that CD $115^{+}$blood monocytes circulating in tumor-bearing mice upregulated several other transcripts that are worthy of mention, such as Cyfip2 which may be a direct target of p53 and may be part of a redundant network of genes responsible for p53-dependent apoptosis (48). Additionally, mRNAs for CX3CL1 (fractalkine) and its receptor CX3CR1 which is generally expressed at low levels on the surface of inflammatory monocytes $(49,50)$ were also increased in monocytes from tumor bearers.

It is interesting to note that blood monocytes from tumor bearers upregulate iNOS2 gene expression yet downregulate NO production. Our gene expression studies were conducted after $2 \mathrm{~h}$ of activation with LPS whereas NO experiments were carried out after $48 \mathrm{~h}$ of LPS activation. Kinetics of constitutive or induced protein expression may vary over time and initial increased transcriptional expression may ultimately result in protein downregulation due to posttranscriptional or posttranslational mechanisms. Our results in NO downregulation in monocytes from tumor bearing mice match with our previously published results in peritoneal macrophages from tumor bearers, which also show a decrease in NO production upon LPS activation; interestingly monocytes from normal mice constitutively produce $\mathrm{NO}$, in contrast to our results in peritoneal macrophages from normal mice which do not produce measurable amounts of NO unless activated by LPS $(13,22)$.

The lack of measurable arginase activity in these cells together with their CD11b bow ${ }^{\text {low }}$ photype indicates that, by definition, they are not MDSCs (51), but instead bona fide monocytes with a mixture of pro-inflammatory and antiinflammatory features.

Immune dysregulation and immune suppression in tumorbearing mice are complex events in which tumor-derived factors condition not only tumor microenvironment and peripheral immune niches, but also the bone marrow, leading to abnormal myelopoiesis and subsequent modification of circulating myeloid cells (25). Tumor cells produce several growth factors and cytokines that stimulate myelopoiesis (GM-CSF, M-CSF, IL-3, VEGF, and IL-6), and it is possible that increased production of these growth factors may affect the normal pathway of cell differentiation resulting in the accumulation of immature monocytes in the blood of tumor-bearing mice (34). The D1-DMBA3 mammary tumor produces GM-CSF and VEGF and high levels of IL-6 are present in the sera of tumor bearers; consequently, blocking these tumor factors may prove effective in reverting tumor-induced immune suppression in monocytes and macrophages from tumor hosts.

\section{Acknowledgements}

We gratefully acknowledge Dr Shannon Opiela for flow cytometry technical support and Dr Diana M. Lopez for critically reading this manuscript. This work was supported by grant K01CA 101926 from the National Institutes of Health (MTK).

\section{References}

1. Balkwill F, Charles KA and Mantovani A: Smoldering and polarized inflammation in the initiation and promotion of malignant disease. Cancer Cell 7: 211-217, 2005.

2. Li H, Fan X and Houghton J: Tumor microenvironment: the role of the tumor stroma in cancer. J Cell Biochem 101: 805-815, 2007.

3. Mantovani A, Sozzani S, Locati M, Allavena P and Sica A: Macrophage polarization: tumor-associated macrophages as a paradigm for polarized M2 mononuclear phagocytes. Trends Immunol 23: 549-555, 2002.

4. Qian BZ and Pollard JW: Macrophage diversity enhances tumor progression and metastasis. Cell 141: 39-51, 2010.

5. Ross JA and Auger MJ: The biology of macrophages. In: The Macrophage. 2nd edition. Burke B and Lewis CE (eds). Oxford University Press, Oxford, 2002.

6. Gordon S and Taylor PR: Monocyte and macrophage heterogeneity. Nat Rev Immunol 5: 953-964, 2005.

7. Auffray C, Sieweke MH and Geissmann F: Blood monocytes: development, heterogeneity, and relationship with dendritic cells. Annu Rev Immunol 27: 669-692, 2009.

8. Geissmann F, Manz MG, Jung S, Sieweke MH, Merad M and Ley K: Development of monocytes, macrophages, and dendritic cells. Science 327: 656-661, 2010.

9. Geissmann F, Auffray C, Palframan R, et al: Blood monocytes: distinct subsets, how they relate to dendritic cells, and their possible roles in the regulation of $\mathrm{T}$-cell responses. Immunol Cell Biol 86: 398-408, 2008.

10. Strauss-Ayali D, Conrad SM and Mosser DM: Monocyte subpopulations and their differentiation patterns during infection. $\mathrm{J}$ Leukoc Biol 82: 244-252, 2007.

11. Yona $S$ and Jung $S$ : Monocytes: subsets, origins, fates and functions. Curr Opin Hematol 17: 53-59, 2010.

12. Geissmann F, Jung S and Littman DR: Blood monocytes consist of two principal subsets with distinct migratory properties. Immunity 19: 71-82, 2003.

13. Torroella-Kouri M, Ma X, Perry G, et al: Diminished expression of transcription factors nuclear factor kappaB and CCAAT/ enhancer binding protein underlies a novel tumor evasion mechanism affecting macrophages of mammary tumor-bearing mice. Cancer Res 65: 10578-10584, 2005.

14. Torroella-Kouri M, Silvera R, Rodriguez D, et al: Identification of a subpopulation of macrophages in mammary tumor-bearing mice that are neither M1 nor M2 and are less differentiated. Cancer Res 69: 4800-4809, 2009.

15. Gallina G, Dolcetti L, Serafini P, et al: Tumors induce a subset of inflammatory monocytes with immunosuppressive activity on $\mathrm{CD}^{+} \mathrm{T}$ cells. J Clin Invest 116: 2777-2790, 2006.

16. Youn JI, Nagaraj S, Collazo M and Gabrilovich DI: Subsets of myeloid-derived suppressor cells in tumor-bearing mice. J Immunol 181: 5791-5802, 2008.

17. Ostrand-Rosenberg S and Sinha P: Myeloid-derived suppressor cells: linking inflammation and cancer. J Immunol 182: 4499-4506, 2009.

18. Medina D and De Ome KB: Response of hyperplastic alveolar nodule outgrowth-line D1 to mammary tumor virus, noduleinducing virus, and prolonged hormonal stimulation acting singly and in combination. J Natl Cancer Inst 42: 303-310, 1969. 
19. Torroella-Kouri M and Lopez DM: Mammary tumor derived TGFb-1 impairs crucial innate immune response in tumor hosts. J Immunol Immunopathol 5: 31-38, 2003.

20. Torroella-Kouri M, Keith JC, Ivanova M and Lopez DM: IL-11induced reduction of $\mathrm{C} / \mathrm{EBP}$ transcription factor binding may contribute to the IL-12 downregulation in tumor-bearing mice. Int J Oncol 22: 439-448, 2003.

21. Stuehr DJ and Nathan CF: Nitric oxide. A macrophage product responsible for cytostasis and respiratory inhibition in tumor target cells. J Exp Med 169: 1543-1555, 1989.

22. Calderon CL, Torroella-Kouri M, Dinapoli MR and Lopez DM: Involvement of protein kinase $\mathrm{C}$ and not of $\mathrm{NF}$ kappa $\mathrm{B}$ in the modulation of macrophage nitric oxide synthase by tumorderived phosphatidyl serine. Int J Oncol 32: 713-721, 2008.

23. De Palma M, Venneri MA, Galli R, et al: Tie2 identifies a hematopoietic lineage of proangiogenic monocytes required for tumor vessel formation and a mesenchymal population of pericyte progenitors. Cancer Cell 8: 211-226, 2005.

24. Lewis CE, De Palma M and Naldini L: Tie2-expressing monocytes and tumor angiogenesis: regulation by hypoxia and angiopoietin-2. Cancer Res 67: 8429-8432, 2007.

25. Sica A and Bronte V: Altered macrophage differentiation and immune dysfunction in tumor development. J Clin Invest 117 : 1155-1166, 2007.

26. Dai XM, Ryan GR, Hapel AJ, et al: Targeted disruption of the mouse colony-stimulating factor 1 receptor gene results in osteopetrosis, mononuclear phagocyte deficiency, increased primitive progenitor cell frequencies, and reproductive defects. Blood 99: 111-120, 2002

27. Hickstein DD, Baker DM, Gollahon KA and Back AL: Identification of the promoter of the myelomonocytic leukocyte integrin CD11b. Proc Natl Acad Sci USA 89: 2105-2109, 1992.

28. Sasmono RT, Oceandy D, Pollard JW, et al: A macrophage colony-stimulating factor receptor-green fluorescent protein transgene is expressed throughout the mononuclear phagocyte system of the mouse. Blood 101: 1155-1163, 2003.

29. Sweet MJ and Hume DA: CSF-1 as a regulator of macrophage activation and immune responses. Arch Immunol Ther Exp (Warsz) 51: 169-177, 2003

30. Auffray C, Fogg D, Garfa M, et al: Monitoring of blood vessels and tissues by a population of monocytes with patrolling behavior. Science 317: 666-670, 2007.

31. Sunderkotter C, Nikolic T, Dillon MJ, et al: Subpopulations of mouse blood monocytes differ in maturation stage and inflammatory response. J Immunol 172: 4410-4417, 2004.

32. Swirski FK, Libby P, Aikawa E, et al: Ly-6Chi monocytes dominate hypercholesterolemia-associated monocytosis and give rise to macrophages in atheromata. J Clin Invest 117: 195-205, 2007

33. Varol C, Yona S and Jung S: Origins and tissue-contextdependent fates of blood monocytes. Immunol Cell Biol 87: 30-38, 2009.

34. Almand B, Clark JI, Nikitina E, et al: Increased production of immature myeloid cells in cancer patients: a mechanism of immunosuppression in cancer. J Immunol 166: 678-689, 2001.
35. Serafini P, Borrello I and Bronte V: Myeloid suppressor cells in cancer: recruitment, phenotype, properties, and mechanisms of immune suppression. Semin Cancer Biol 16: 53-65, 2006.

36. Imhof BA and Aurrand-Lions M: Adhesion mechanisms regulating the migration of monocytes. Nat Rev Immunol 4: 432-444, 2004

37. Venneri MA, De Palma M, Ponzoni M, et al: Identification of proangiogenic TIE2-expressing monocytes (TEMs) in human peripheral blood and cancer. Blood 109: 5276-5285, 2007.

38. Loveland BE and Cebon J: Cancer exploiting complement: a clue or an exception? Nat Immunol 9: 1205-1206, 2008.

39. Markiewski MM, De Angelis RA, Benencia F, et al: Modulation of the antitumor immune response by complement. Nat Immunol 9: 1225-1235, 2008.

40. Markiewski MM and Lambris JD: Unwelcome complement. Cancer Res 69: 6367-6370, 2009.

41. Markiewski MM and Lambris JD: Is complement good or bad for cancer patients? A new perspective on an old dilemma. Trends Immunol 30: 286-292, 2009.

42. Dolcetti L, Marigo I, Mantelli B, Peranzoni E, Zanovello P and Bronte V: Myeloid-derived suppressor cell role in tumor-related inflammation. Cancer Lett 267: 216-225, 2008.

43. Melgarejo E, Medina MA, Sanchez-Jimenez F and Urdiales JL: Monocyte chemoattractant protein-1: a key mediator in inflammatory processes. Int J Biochem Cell Biol 41: 998-1001, 2009.

44. Tacke F, Alvarez D, Kaplan TJ, et al: Monocyte subsets differentially employ CCR2, CCR5, and CX3CR1 to accumulate within atherosclerotic plaques. J Clin Invest 117: 185-194, 2007.

45. Tsou CL, Peters W, Si Y, et al: Critical roles for CCR2 and MCP-3 in monocyte mobilization from bone marrow and recruitment to inflammatory sites. J Clin Invest 117: 902-909, 2007.

46. Schioppa T, Uranchimeg B, Saccani A, et al: Regulation of the chemokine receptor CXCR4 by hypoxia. J Exp Med 198: 1391-1402, 2003

47. Mantovani A, Allavena P and Sica A: Tumour-associated macrophages as a prototypic type II polarised phagocyte population: role in tumour progression. Eur J Cancer 40: 1660-1667, 2004.

48. Jackson RS II, Cho YJ, Stein S and Liang P: CYFIP2, a direct p53 target, is leptomycin-B sensitive. Cell Cycle 6: 95-103, 2007.

49. Li L, Huang L, Sung SS, et al: The chemokine receptors CCR2 and CX3CR1 mediate monocyte/macrophage trafficking in kidney ischemia-reperfusion injury. Kidney Int 74: 1526-1537, 2008.

50. Serbina NV and Pamer EG: Monocyte emigration from bone marrow during bacterial infection requires signals mediated by chemokine receptor CCR2. Nat Immunol 7: 311-317, 2006.

51. Bronte V and Zanovello P: Regulation of immune responses by L-arginine metabolism. Nat Rev Immunol 5: 641-654, 2005. 